\title{
Multi-Dimensional Discrete Stochastic Calculus and \\ its application to a problem in mathematical finance
}

\author{
Jirô Akahori \\ Research Center for Finance/Department of Mathematical Sciences \\ Ritsumeikan University \\ 1-1-1, Nojihigashi, Kusatsu, Shiga, 525-8577, Japan \\ akahori@se.ritsumei.ac.jp
}

\begin{abstract}
The present paper gives a brief survey of discrete Itô calcullus, which was recently introduced by the author. An application of the calculus to a multi-dimensional version of CoxRoss-Rubinstein model will be presented.
\end{abstract}

\section{Introduction}

The stochastic calculus, which is often cited with the name of its founder, K. Itô, is no doubt among the most successfully applied mathematics; it is applied to physics, engineering, and, most recently, to finance. Basically, the Itô's stochastic calculus is a calculus for Brownian motions, equipped with a formula for differentiations of composite functions; Itô's formula.

As is well known, a Brownian motion is approximated by a sum of independent random variables. In such discrete settings, however, it seems that one has been missing the corresponding differential formula. But in fact, discrete versions of Itô's formula do exists. Historically, a version of discrete Itô's formula was introduced in late 80's [8] by Prof. Tamas Szabados, a Hungarian probabilist. Recently, Prof. Takahiko Fujita [6] introduced a version that gives Doob decomposition of the process which is described by a function of the simple random walk. This version made a significant progress since it relates an expectation of the random walk to a difference equation which comes from the predictable part of the formula. This viewpoint is developed by the author in [1].

We will introduce in the following section a multi-dimensional random walk that has martingale representation property and then in section 3, a discrete Itô's formula. These results are, as a whole, borrowed from such papers as [1], [2], [3], [6], and [8]. And then, in section 4, an application to a multi- 
dimensional version of Cox-Ross-Rubinstein (CRR for short) [5] model will be discussed. The topic is original of the present paper, though it can be seen as a special case of the ones treated in [1].

\section{The Sum of Random Phases}

Let $n \geq 2$ be an integer and let $\tau$ be a random variable taking values in $C_{n+1}:=\left\{x \mid x^{n+1}=\right.$ $1\}=\left\{e^{2 \pi i k /(n+1)}: k=0,1, \ldots, n\right\}$ with equal probability. We may call $\tau$ random phase, which is a natural extension of the random sign (the case when $n=1$ ). Let $\tau_{1}, \tau_{2}, \ldots$ be independent copies of $\tau$. For $i=1, \ldots, n$ and $z=\left(z_{1}, \ldots, z_{n}\right) \in \mathbf{C}^{n}$ put

$$
Z_{k}^{i}=z^{i}+\tau_{1}^{i}+\cdots+\tau_{k}^{i},(k \in \mathbf{N}) .
$$

Each $Z^{i}$ is regarded as a natural extension of the simple symmetric random walk (= the sum of random signs), taking values in the ring of algebraic integers $\mathbf{Z}\left[e^{2 \pi i /(n+1)}\right]$ (while the sum of random signs takes its values in the ring of integers in the usual sense).

Let $\phi: \mathbf{C} \rightarrow \mathbb{R}$ defined by $\phi(z)=\operatorname{Re}(z)+$ $\operatorname{Im}(z)$. The $n$-dimensional random walk $W$ defined by

$$
W_{t} \equiv\left(W_{t}^{1}, \ldots, W_{t}^{n}\right):=\left(\phi\left(Z_{t}^{1}\right), \ldots, \phi\left(Z_{t}^{n}\right)\right),
$$

plays a central role in our discrete Itô calculus (stochastic calculus). This is because

- it has the martingale representation property and just like $n$-dimensional Brownian motions, its multiplicity is $n$.

- it approximates $n$-dimensional Brownian motions; after taking proper scaling, its law converges to the $n$-dimensional Wiener measure.
To be precise, we have the following results (For details, see [3]).

Theorem 1 ([3]). Set $\mathcal{F}_{n}:=\sigma\left(\tau_{1}, \ldots, \tau_{n}\right)$ for $n \in \mathbf{N}$ and let $\mathbf{F}$ be the filtration composed of $\mathcal{F}_{n}$ 's. Then every square integrable $\mathcal{F}$. martingale is represented as a stochastic integral with respect to $W$. More precisely, we have the following.

For each $\mathbf{F}$-adapted process $\left\{M_{t}\right\}$ with

$$
\sup _{t} \mathbb{E}\left[\left|M_{t}\right|^{2}\right]<\infty,
$$

there exists an $\mathbf{R}^{n+1}$ valued $\mathbf{F}$-predictable process

$$
\left\{\theta_{t}=\left(\theta_{t}^{0}, \theta_{t}^{1}, \ldots, \theta_{t}^{n}\right)\right\}
$$

with

$$
\sum_{t=1}^{\infty} \sum_{i=0}^{n}\left|\theta_{t}^{i}\right|^{2}<\infty
$$

such that for every $t \in \mathbf{N}$,

$$
M_{t}=\sum_{u=1}^{t}\left(\sum_{i=1}^{n} \theta_{u}^{i}\left(W_{u}^{i}-W_{u-1}^{i}\right)+\theta_{u}^{0}\right) .
$$

Theorem 2 ([3]). Let $T \in(0, \infty)$ and put $\Delta:=$ $T / N$. For each $N \in \mathbf{N}$, define

$$
\mathbf{W}_{t}^{N}:=(\sqrt{\Delta})\left(W_{[t / \Delta]}^{1}, \ldots, W_{[t / \Delta]}^{n}\right): t \in[0, T] .
$$

Then the law of $\mathrm{W}^{N}$ converges to the $n$ dimensional Wiener measure on $C[0, T]$ as $N \rightarrow \infty$.

These theorems are almost direct consequences of Schur's orthogonality of characters of the cyclic group and the martingale central limit theorem. 


\section{A Discrete Itô's formula}

Thanks to the above theorems, we can prove a discrete version of Itô's formula which we call Szabados-Fujita formula. Below we present the formulas for (i) $Z=\left(Z^{1}, \ldots, Z^{n}\right)$ and (ii) $W^{N}=\left(W^{1, N}, \ldots, W^{n, N}\right)$.

Theorem 3 ([8], [6], [1], [2], [3]). (i) For arbitrary $f:\left(\mathbb{Z}\left[e^{2 \pi i /(n+1)}\right]\right)^{n} \rightarrow \mathbf{C}$, we have

$$
\begin{aligned}
& f\left(Z_{t+1}\right)-f\left(Z_{t}\right) \\
& =\sum_{i=1}^{n} D_{i} f\left(Z_{t}\right)\left(Z_{t+1}^{i}-Z_{t}^{i}\right)+L f\left(Z_{t}\right),
\end{aligned}
$$

where $D_{i}$ and $L$ are difference operators defined by

$$
\begin{array}{r}
D_{i} f(z)=\frac{1}{n+1} \sum_{\zeta \in C_{n+1}} f(z+\vec{\zeta}) \zeta^{n+1-i} \\
(i=1,2, \ldots, n), \\
L f(z)=\frac{1}{n+1} \sum_{\zeta \in C_{n+1}}\{f(z+\vec{\zeta})-f(z)\} .
\end{array}
$$

Here we write

$$
\vec{\zeta}=\left(\zeta, \zeta^{2}, \ldots, \zeta^{n}\right) \in\left(\mathbb{Z}\left[e^{2 \pi i /(n+1)}\right]\right)^{n}
$$

(ii) For $f:[0, \infty) \times \mathbf{R}^{n} \rightarrow \mathbf{R}$, we have

$$
\begin{aligned}
& f\left(t, W_{t}^{N}\right)-f\left(0, W_{0}^{N}\right) \\
& =\sum_{k=1}^{[t / \Delta]}\left\{\sum_{i=1}^{n} \partial_{i}^{N} f\left(k \Delta, W_{(k-1) \Delta}^{N}\right)\left(\Delta W_{k \Delta}^{i, N}\right)\right. \\
& \left.\left.\quad+\left(\mathcal{L}^{N}+\partial_{t}^{N}\right) f\left(k \Delta, W_{(k-1) \Delta}^{N}\right)\right\} \Delta\right\},
\end{aligned}
$$
where $\left(\Delta W_{u}^{i, N}\right):=W_{u}^{i, N}-W_{u-\Delta}^{i, N}$, and $\partial_{i}^{N}, \mathcal{L}^{N}$, and [3].

$$
\begin{aligned}
& \partial_{i}^{N} f(\cdot, x) \\
& =\frac{1}{(n+1) \sqrt{\Delta}} \sum_{\zeta \in C_{n+1}} f(\cdot, x+\sqrt{\Delta} \phi \overrightarrow{(\zeta)}) \phi\left(\zeta^{i}\right) \\
& \mathcal{L}^{N} f(\cdot, x) \\
& =\frac{1}{(n+1) \Delta} \sum_{\zeta \in C_{n+1}}\{f(\cdot, x+\sqrt{\Delta} \phi(\vec{\zeta}))-f(\cdot, x)\} \\
& \partial_{t}^{N} f(u, \cdot) \\
& =\{f(u, \cdot)-f(u-\Delta)\} / \Delta
\end{aligned}
$$

Here we write

$$
\phi \overrightarrow{(\zeta)}=\left(\phi(\zeta), \phi\left(\zeta^{2}\right), \ldots, \phi\left(\zeta^{n}\right)\right)
$$

It should be noted that the operators in (ii) converges to corresponding differential operators as $N \rightarrow \infty$. Detailed studies about the order of convergence are found in [1] as

Theorem 4 ([1]). If $f \in C^{1,3}(U)$ in an open set of $[0, T] \times \mathbf{R}^{n}$, then for arbitrary compact set $K \subset U$, there exists a positive constant $C_{K}$ such that

$$
\begin{aligned}
\max _{i,(u, x) \in K}\left(\left|\partial_{i}^{N} f(u, x)-\partial_{i} f(u, x)\right|,\right. \\
\\
\left|\mathcal{L}^{N} f(u, x)-\mathcal{L} f(u, x)\right|, \\
\left.\left|\partial_{t}^{N} f(u, x)-\partial_{t}(u, x)\right|\right)<C_{K} n^{-1 / 2} .
\end{aligned}
$$

Here $\partial_{i} f$ 's and $\partial_{t} f$ denote the usual partial derivatives, and $\mathcal{L}$ denotes the $n$-dimensional Laplacian.

These results have several applications, but we will present only one example in the next section. Other applications are reported in [1] 


\section{An Application to Math- ematical Finance; Multi- Dimensional CRR}

As is well known, the Black-Scholes economy [4] (where only one stock and one non-risky bond are traded; the stock price is assumed to follow the geometric Brownian motion and the return rate of the bond is constant.) is approximated by a sequence of economy generated by the sum of random signs. This is known as CRR procedure.

Here we extend CRR to a multidimensional setting within He's framework [7], following the results in [1].

Let $N, T$ and $\Delta$ be as in the preceding sections. Suppose that there are $n$ risky assets whose price at $k$-th trading date $(k \in$ $\{1,2, . ., N\})$ are described by

$$
\begin{aligned}
& S_{k \Delta}^{i, N} \quad(i=1, \ldots, n) \\
& =S_{0}^{i} \exp \left(\sum_{j=1}^{n} \sigma_{i j} W_{k \Delta}^{j, N}+\left(\mu_{i}-\sum_{j=1}^{n} \sigma_{i j}^{2} / 2\right) k \Delta\right),
\end{aligned}
$$

and there is one non-risky asset whose price at $k$ is described as

$$
S_{k \Delta}^{0, N}=\exp (r k \Delta)
$$

where $S_{0}^{i}$ for $i=1, \ldots, n$ are given as initial values, $\sigma_{i j}$ 's and $r$ are positive constants, and $\mu_{i}$ are real constants that indicate the (asymptotic) expected returns. With a continuous limit in mind, we may assume that $\Sigma:=$ $\left(\sigma_{i j}\right)=\mathcal{V}^{1 / 2}$, where $\mathcal{V}$ is a given covariance matrix for the returns, and we may also assume that $\Sigma>0$.

The discounted price processes for $i=$ $1, \ldots, n$ are described as

$$
\widetilde{S}_{k \Delta}^{i, N}=S_{0}^{i} \exp \left\{\sum_{j=1}^{n} \sigma_{j} W_{k \Delta}^{j, N}+\left(\widetilde{\mu}_{i} k \Delta\right)\right\},
$$

where we have set

$$
\widetilde{\mu}_{i}:=\mu-r-\sum_{j=1}^{n} \sigma_{i j}^{2} / 2 .
$$

Define $f^{i, N} \in C^{\infty}\left([0, T] \times \mathbb{R}^{n} \rightarrow \mathbb{R}\right)$ for $i=$ $1, \ldots, n$ by

$$
f^{i}\left(t, x_{1}, \ldots, x_{n}\right)=S_{0}^{i} \exp \left\{\sum_{j=1}^{n} \sigma_{i j} x_{j}+\left(\tilde{\mu}_{i} t\right)\right\} .
$$

Then we have $\widetilde{S}_{t}^{i, N}=f^{i, N}\left(t, W_{t}^{N}\right)$ for $t=$ $\Delta, 2 \Delta, \ldots, T=N \Delta$, and by the Szabados-Fujita formula (Theorem 3 (ii)), we have

$$
\begin{aligned}
& \left(\Delta \widetilde{S}^{i, N}\right)_{k \Delta}:=\widetilde{S}_{k \Delta}^{i, N}-\widetilde{S}_{(k-1) \Delta}^{i, N} \\
& =\sum_{j=1}^{n} \partial_{j} f^{i}\left(k \Delta, W_{(k-1) \Delta}^{N}\right)\left(\Delta W^{j, N}\right)_{k \Delta} \\
& \quad+\left(\mathcal{L}^{N}+\partial_{t}^{N}\right) f^{i}\left(k \Delta, W_{(k-1) \Delta}^{N}\right) \Delta .
\end{aligned}
$$

Here we have explicitly

$$
\begin{aligned}
& \partial_{j} f^{i}(t, x) \\
& =\frac{f^{i}(t, x)}{(n+1) \sqrt{\Delta}} \sum_{\zeta \in C_{n+1}} e^{\sqrt{\Delta} \sum_{i} \phi\left(\zeta^{i}\right)} \phi\left(\zeta^{j}\right) \\
& =: \sigma_{i j} f^{i}(t, x)\left(1+\epsilon_{i j}(\sqrt{\Delta})\right), \\
& \mathcal{L}^{N} f^{i}(t, x) \\
& =\frac{f^{i}(t, x)}{(n+1) \Delta} \sum_{\zeta \in C_{n+1}}\left\{e^{\sqrt{\Delta} \sum_{i} \phi\left(\zeta^{i}\right)}-1\right\} \\
& =: \frac{\sum_{j} \sigma_{i j}^{2}}{2} f^{i}(t, x)\left(1+\epsilon_{L}(\sqrt{\Delta})\right),
\end{aligned}
$$

and

$$
\begin{aligned}
\partial_{t} f^{i}(t, x) & =\frac{f^{i}(t, x)}{\Delta}\left(1-e^{-\bar{\mu}_{i} \Delta}\right) \\
& =: \tilde{\mu}_{i} f^{i}(t, x)\left(1-\epsilon_{t}(\Delta)\right) .
\end{aligned}
$$

Note that all $\epsilon .(x)$ defined here are in an order of $\mathrm{O}(x)$; there exists a constant independent of $x$ such that

$$
\epsilon .(x) \leq C x \text {. }
$$


Suppose that we have an European contingent claim whose pay-off at the maturity date $T$ is $H\left(W_{T}^{1, N}, \ldots, W_{T}^{n, N}\right)$ for some bounded measurable $H: \mathbb{R}^{n} \rightarrow \mathbf{R}$. If we can represent

$$
\begin{aligned}
H\left(W_{T}^{1, N}, \ldots,\right. & \left.W_{T}^{n, N}\right) / S_{T}^{0, N} \\
& =H_{0}^{N}+\sum_{k=1}^{n} \eta_{(k-1) \Delta}^{i, N}\left(\Delta \widetilde{S}^{i, N}\right)_{k \Delta,}
\end{aligned}
$$

by some adapted $\eta=\left(\eta^{1}, \ldots, \eta^{n}\right)$ and a constant $H_{0}$, we can safely say that the price is $H_{0}$, and the hedging strategy is just $\eta$, by the standard no-arbitrage argument.

This can be done by the following theorem. Before stating the result we prepare some notations:

$$
\begin{aligned}
\Sigma_{N} & :=\left(\sigma_{i j}(1+\epsilon(\sqrt{\Delta}))\right. \\
\nabla^{N} & :=\left(\partial_{1}^{N}, \ldots, \partial_{n}^{N}\right),
\end{aligned}
$$

and

$$
\vec{\mu}^{N}:=\left(\mu_{1}^{N}, \ldots, \mu_{n}^{N}\right),
$$

where

$$
\mu_{i}^{N}:=\widetilde{\mu}_{i}\left(1-\epsilon_{t}(\Delta)\right)+\frac{\sum_{j} \sigma_{i j}^{2}}{2}(1+\epsilon(\sqrt{\Delta})) .
$$

Note that $\Sigma_{N} \rightarrow \Sigma, \nabla^{N} \rightarrow \nabla$ in an appropriate sense, and $\mu_{i}^{N} \rightarrow \mu_{i}-r$. We also denote by $\mathcal{X}_{k}^{x, N}$ the set of reachable point of $W_{k \Delta}^{N}$ which starts $x \in \mathbb{R}^{n}$.

Now we are ready to state

Theorem 5. Consider the following difference equation:

$$
\left(\partial_{t}^{N}+L^{N}-\left\langle\Sigma_{N}^{-1} \vec{\mu}^{N}, \nabla^{N}\right\rangle\right) u(t, x)=0
$$

for $t=\Delta, 2 \Delta, \ldots, n \Delta=T$ and $x \in \mathcal{X}_{t / \Delta}^{0, N}$, with boundary condition

$$
u(T, x)=H(x), \quad x \in \mathcal{X}_{N}^{0, N} .
$$

Then for sufficiently large $N$, it has a unique solution $u^{N}$ and we have (2) by setting

$$
H_{0}^{N}=u^{N}(0,0)
$$

and $\eta_{t}^{i, N}=\tilde{\eta}_{t}^{i, N} / \tilde{S}_{t}^{i, N}$ where

$$
\tilde{\eta}_{t}^{N} \equiv\left(\tilde{\eta}_{t}^{1, N}, \ldots, \tilde{\eta}_{t}^{1, N}\right)=\Sigma_{N}^{-1} \nabla^{N} u^{N} .
$$

Proof. Suppose that the solution $u^{N}$ exists. By the Szabados-Fujita formula, we have

$$
\begin{aligned}
& u^{N}\left(n \Delta, W_{t}^{N}\right)-u^{N}\left(0, W_{0}^{N}\right) \\
& =\sum_{k=1}^{l}\left\{\sum_{i=1}^{n} \partial_{i}^{N} u^{N}\left(k \Delta, W_{(k-1) \Delta}^{N}\right)\left(\Delta W_{k \Delta}^{i, N}\right)\right. \\
& \left.\left.+\left(\mathcal{L}^{N}+\partial_{t}^{N}\right) u^{N}\left(k \Delta, W_{(k-1) \Delta}^{N}\right)\right\} \Delta\right\} \\
& =\sum_{k=1}^{n}\left\{\left\langle\nabla^{N} u^{N}\left(k \Delta, W_{(k-1) \Delta}^{N}\right),\left(\Delta W_{k \Delta}^{N}\right)\right\rangle\right. \\
& \left.\quad+\left\langle\Sigma_{N}^{-1} \vec{\mu}^{N}, \nabla^{N} u^{N}\left(k \Delta, W_{(k-1) \Delta}^{N}\right)\right\rangle \Delta\right\},
\end{aligned}
$$

where we have set

$$
\left(\Delta W_{k \Delta}^{N}\right):=\left(\left(\Delta W_{k \Delta}^{1, N}\right), \ldots,\left(\Delta W_{k \Delta}^{n, N}\right)\right) .
$$

On the other hand, a closer look of (1) leads to

$$
\left(\Delta W_{k \Delta}^{N}\right)=\Sigma_{N}^{-1}\left(\left(\Delta \widetilde{S}^{N} / \widetilde{S}^{N}\right)_{k \Delta}-\vec{\mu}^{N} \Delta\right),
$$

where we have set

$$
\left(\Delta \widetilde{S}^{N} / \widetilde{S}^{N}\right)_{t}=\left(\frac{\left(\Delta \widetilde{S}^{1, N}\right)_{t}}{\widetilde{S}_{t-\Delta}^{1, N}}, \ldots, \frac{\left(\Delta \widetilde{S}^{n, N}\right)_{t}}{\widetilde{S}_{t-\Delta}^{n, N}}\right) .
$$

Substituting (5) into (4), we get (2).

Next we will show the existence of the solution. As a matter of course, $\Sigma_{N}^{-1}$ exists for sufficiently large $N$, and the equation (3) can be rewritten as

$$
\begin{aligned}
& u((k-1) \Delta, x)=u(k \Delta, x) \\
& +\left\{L^{N}-\left\langle\Sigma_{N}^{-1} \vec{\mu}^{N}, \nabla^{N}\right\rangle\right\} u(k \Delta, x),
\end{aligned}
$$

for $k=1,2, \ldots, n$. This means that the equation (3) is nothing but a backward recursive equation. Thus a unique solution always exists. 
By theorem 4, we can prove that $u_{N}$ converges to a continuous limit $u \in C^{1,3}$ (if it exists) in an order of $O(\sqrt{\Delta})$. This result is a special case of a result found in [1]. One can easily check that if

$$
\begin{aligned}
& H\left(x_{1}, \ldots, x_{n}\right) \\
& \quad=\tilde{H}\left(f^{1}(T, x), f^{2}(T, x), \ldots, f^{n}(T, x)\right),
\end{aligned}
$$

(regarding $T$ as a constant) for some $H$ : $\left(\mathbf{R}_{>0}\right)^{n} \rightarrow \mathbf{R}$, the solution do not depends on $\mu_{i}$ 's but depends on $S_{0}^{i}$ 's. This recovers the well-known implication of the original BlackScholes formula.

Remark 6. The right-hand-side of (6) is actually a linear combination of $u(k \Delta, x+$ $\phi(\vec{\zeta})) / S_{k \Delta}^{0}$. The coefficients corresponds to the risk neutral probability of $\tau_{k \Delta}=\zeta$, provided that they are all strictly positive. The existence of equivalent martingale measure, which is equivalent to the non-existence of arbitrage opportunities, can be judged from those coefficients. For details, see [1].

\section{References}

[1] Akahori, J. "A discrete Itô calculus approach to He's framework for multi-factor discrete markets." preprint, 2003.

[2] Akahori. J. "Local time in Parisian walkways." preprint, 2003.

[3] Akahori, J., Aoki,H. and Nagata, Y. "A general framework for finite-factor discrete-time interest rate models." preprint, 2002.

[4] Black, F., and M. Scholes. "The Pricing of Options and Corporate Liability." Journal of Political Economics, 81 pp. 637-654, 1973.
[5] Cox, J.,Ross, S.A. and Rubinstein, M. "Option pricing: a simple approach." Journal of Financial Economics, 7, 229-263, 1979.

[6] Fujita, T. "A random walk analogue of Lévy's theorem." preprint, 2003.

[7] He, H. "Convergence from discrete- to continuous-time contingent claim prices." The Review of Financial Studies, 3, 523546, 1990.

[8] Szabados, T. "A discrete Ito's formula. Limit theorems in probability and statistics." Colloq. Math. Soc. Janos Bolyai, 57, 491-502, 1989. 